\title{
Le Brésil de Lula : comment consolider la démocratie en Amérique latine?
}

Entretien conduit par Jean-Luc Racine

Brazil under Lula: How to consolidate democracy in Latin America?

\section{Hélgio Trindade}

\section{OpenEdition}

\section{Journals}

Édition électronique

URL : http://journals.openedition.org/transcontinentales/653

DOI : 10.4000/transcontinentales.653

ISBN : 978-2-7351-1565-5

ISSN : 1775-397X

\section{Éditeur}

Editions de la maison des sciences de l'homme

\section{Édition imprimée}

Date de publication : 1 juin 2007

Pagination : 121-129

ISBN : 978-2-200-92396-9

ISSN : 1950-1684

\section{Référence électronique}

Hélgio Trindade, "Le Brésil de Lula : comment consolider la démocratie en Amérique latine? », Transcontinentales [En ligne], 4 | 2007, document 7, mis en ligne le 28 avril 2011, consulté le 08 septembre 2020. URL : http://journals.openedition.org/transcontinentales/653 ; DOI : https://doi.org/ 10.4000/transcontinentales.653 


\title{
Entretien avec Hélgio Trindade
}

\section{Le Brésil de Lula : comment consolider la démocratie en Amérique latine?}

\begin{abstract}
Hélgio Trindade est professeur de sciences politiques à l'université fédérale du Rio Grande do Sul, à Porto Alegre, université dont il a été recteur. Il a travaillé sur les mouvements autoritaires (La tentation fasciste au Brésil dans les années trente, Éditions de la MSH, Paris, 1988) et plus largement sur les idéologies politiques au Brésil. Président de la commission nationale d'évaluation de l'enseignement supérieur, il dirige aujourd'hui des recherches sur la genèse et sur l'état des sciences sociales en Amérique latine (dossier in Social Sciences Information, vol. 44, n²-3, 2005). Membre de l'Académie des sciences du Brésil, il siège au conseil scientifique de Transcontinentales.
\end{abstract}

Populismes, néolibéralisme, régimes militaires, virage à gauche, social-démocratie et radicalisme : dans le cadre d'un large panorama de l'Amérique latine depuis les années 1930 Hélgio Trindade dessine le nouveau profil du Brésil de Lula, qui entend concilier croissance économique, stabilité financière et politique sociale, pour consolider la démocratie.

Transcontinentales: L'Amérique latine vire à gauche. En 2005 et 2006, neuf élections présidentielles ont vu la victoire des candidats de gauche, et dans deux cas notables, au Brésil et en Bolivie, leur réélection. Faut-il interpréter ces résultats comme un mouvement d'ensemble, marquant un retour de balancier après les choix ultra-libéraux des années 1980-1990, ou faut-il souligner plutôt le fait que ce mouvement recouvre de grandes différences dans les idéologies, comme dans les rapports de force politique: de la "révolution bolivarienne» d'Hugo Chávez, par exemple, à la social-démocratie de Michelle Bachelet qui gouverne avec des démocrates-chrétiens?

Hélgio Trindade : Aujourd'hui, le sens de deux concepts - "gauche» et "populisme» - doit être précisé dans le contexte latino-américain. Celui du populisme recouvre en effet des réalités très différentes. Après les populismes classiques des années 1930 - le péronisme en Argentine et le varguisme au Brésil - le mot réapparut pour qualifier des gouvernements néo-libéraux, tels que ceux de Carlos Menem en Argentine (1989-1999) et d'Alberto Fujimori au Pérou (1990-2000), alors qu'en Europe le populisme désigne aussi bien la droite italienne de Silvio Berlusconi que l'extrême droite française de Jean-Marie Le Pen. Quant au concept de gauche, il a lui aussi évolué : il est moins univoque qu'avant la chute du mur de Berlin. Dans cette perspective, dire que l'Amérique latine "vire à gauche» suppose que les points communs l'emportent sur les différences entre ces gouvernements. 
D'un côté, Michelle Bachelet, au Chili, est plus proche de la social-démocratie européenne alliée à un parti démocrate-chrétien moins conservateur que ceux d'Europe, dans un pays traditionnellement structuré autour d'une droite libérale et d'une gauche socialiste et communiste. La stratégie de la "Concertation des partis pour la démocratie» de Bachelet cherche à fonder une alliance stable du centre gauche, sans les communistes. À l'autre extrême, se rangent les gouvernements d'Hugo Chávez au Venezuela et d'Evo Morales en Bolivie, qui incarnent une gauche nationaliste et révolutionnaire, dans des États confrontés à des problèmes économiques et sociaux comparables à ceux que l'Argentine et le Brésil ont connus dans les années 1940-1950.

En situation intermédiaire, le Brésil dispose d'un système de partis structurellement dominé par des formations libérales-conservatrices, plus proches du pragmatisme des partis américains que de la tradition européenne, plus idéologique. Les partis de la gauche communiste et socialiste sont historiquement faibles, sauf le Parti travailliste brésilien (PTB), qui devint majoritaire avec João Goulart, élu à la présidence de la République en 1961 avant d'être renversé par un coup d'État en 1964. Fondé en 1945, et lié au populisme de Vargas, le PTB dut son ascension au processus d'urbanisation et au syndicalisme développé depuis la Révolution de 1930, qui lui permirent de devenir la base du «national-populisme» brésilien dans les années 1950-1964. Après le suicide de Vargas en 1954, les héritiers du travaillisme (Goulart et Brizola) furent écartés du pouvoir par les militaires et par l’opposition libérale-démocratique (l’Union démocratique nationale) qui fit alliance avec les militaires au pouvoir. En 1980, cinq ans avant la fin du régime militaire, le nouveau Parti des travailleurs fut fondé, à partir des mouvements syndicaux de São Paulo. Il fallut attendre 2002 pour le voir porter Lula à la présidence de la République après trois défaites successives face à des présidents d'orientation néo-libérale : il s'agit bien alors d'un "virage à gauche», dans un contexte plus conservateur, mais qui n'a pas de liens avec le populisme d'antan.

Quant à Nestor Kirchner, président de l'Argentine depuis 2003, s'il vient d'une filiation péroniste comme Carlos Menem, il tient un discours plus à gauche que celui de Lula et conduit une politique plus radicale en matière du remboursement des emprunts de son pays auprès des organismes internationaux, non sans succès dans la gestion de la crise économique et financière. Son ambition est de faire la synthèse entre les deux mouvements historiques du $\mathrm{xx}^{e}$ siècle - le «radicalisme» et le "péronisme»-, mais les marges de manœuvre sont limitées, comme au Brésil, par les politiques fiscales, et par un mode de croissance économique qui doit beaucoup aux exportations.

Il manque une stratégie de long terme pour les deux pays, mais le Brésil cherche à aller plus avant par des politiques gouvernementales coordonnées, tel le «Plan d'accélération de la croissance» (PAC) lancé en 2007. À l’inverse des politiques néo-libérales qu'ont connues l'Argentine, le Brésil et le Mexique, la tendance en Amérique latine aujourd'hui est de revaloriser l'action étatique dans le domaine économique et social. Ce qu'explique en partie le succès du paradoxe chilien: 
au Chili, malgré les politiques des "Chicago boys ${ }^{1}$ ", l'État a préservé son rôle de coordination économique. D’Allende à Bachelet, en passant par la dictature de Pinochet, l'État chilien n'a pas privatisé le cuivre, par exemple.

Au total, ce qui distingue ces gouvernements élus depuis quelques années de leurs prédécesseurs tient à leur ancrage à la gauche lato sensu et à leur très large base électorale. Ils ont en commun une idéologie opposée à l'hégémonie néo-libérale des années 1990.

TC : Comment s'inscrit aujourd'hui le populisme dans la diversité des situations latino-américaine? Populisme de droite ou de gauche, faut-il y voir une forme immature de la démocratie où le rapport primordial relie l'électorat à un leader charismatique, faute de structures assez efficaces pour faire entendre des voix diverses par de multiples canaux (associations, syndicats, médias, etc.) atténuant la tentation de la personnalisation du pouvoir?

H.T. : Par référence à l'époque du populisme sud-américain classique (Perón et Vargas) ou à la personnalisation du pouvoir dans la conjoncture internationale de la guerre froide (de Gaulle, Khrouchtchev ou Castro), certains analystes ont tendance à reprendre ces concepts pour expliquer la nouvelle conjoncture politique dans certains pays de l'Amérique latine. Cette démarche est contestable. Mettre en avant le populisme aujourd'hui tend à appauvrir la richesse des changements qui définissent l'apport actuel de l'Amérique latine à la politique mondiale. L'impact international de l'envolée économique de la Chine a renforcé la nécessité d'un polycentrisme, dont les nouveaux leaderships latino-américains sont une manifestation. Les gouvernements latino-américains actuels ne doivent plus être considérés "en bloc», car la plupart des pays ont défini leur propre stratégie régionale et internationale. Leurs sociétés sont devenues plus complexes, plus organisées. La démocratie se consolide dans la plupart de ces pays, sans risque d'un retour des militaires, avec la présence de mouvements populaires et sociaux comme le Mouvement sans terre au Brésil ou les piqueteros en Argentine ${ }^{2}$.

TC : Vous êtes professeur de sciences politiques à Porto Alegre, la ville qui a été le terrain d'expérimentation de la démocratie participative, et le foyer où se sont tenues, de 2001 à 2003, les premières rencontres du Forum social mondial. Qu'a représenté pour vous cette expérience, née du mouvement social brésilien, puis étendue à toute la planète? La genèse du Forum social mondial a aidé à la construction du mouvement altermondialiste. Comment évaluer aujourd'hui ce mouvement, au Brésil et en Amérique latine, alors que l'expérience du premier mandat de Lula a été plutôt marquée par un réformisme mesuré et pragmatique?

1 - Sous Pinochet, on appelait «Chicago boys» un groupe d'économistes très influents - beaucoup furent ministres - partisans de la libéralisation du marché. Ils sortaient de l'École d'économie de l'université catholique du Chili, qui mit en œuvre, à compter de 1956, un accord avec l'université de Chicago, dont des professeurs vinrent enseigner au Chili. Milton Friedman en fut l'un des maîtres à penser. (NDLR)

2 - On appelle piqueteros en Argentine les militants des mouvements de protestation, lancés au départ par des chômeurs, et qui dressaient des barrages sur les routes. (NDLR) 
H.T. : Assurément, la présence des élus du Parti des travailleurs pendant seize ans à la mairie de Porto Alegre a permis la mise au point des «budgets participatifs» comme expérience réussie de démocratie locale. S'est développé ensuite un espace nouveau pour les mouvements altermondialistes, à travers le Forum social mondial. Ces processus ont joué à l'époque un rôle important, celui d'expériences alternatives à la démocratie représentative. Ils sont devenus comme une image de marque de Porto Alegre pour d'autres régions du pays, puis une facette emblématique du Brésil sur le plan international.

Mais l'accession du Parti des travailleurs à des gouvernements d'États puis au pouvoir national a deplacé l'axe politique des avancées démocratiques, au-delà du local. À l'origine, le Forum social mondial (FSM) doit beaucoup au pouvoir conquis par le Parti des travailleurs à Porto Alegre, considérée comme la «ville rouge» dans une période qui était marquée par l'hégémonie du discours néolibéral dans l'État du Rio Grande do Sul, au niveau national, et dans nombre de pays latino-américain. Mais après la défaite du Parti des travailleurs aux élections municipales en 2004, le Forum a perdu une partie de sa force effective et de son aura symbolique à Porto Alegre. Avant même cette défaite, le Conseil international du Forum social mondial s'était prononcé pour une stratégie de diversification de son implantation. Alors même que le Parti des travailleurs était toujours en place à la mairie de Porto Alegre, le Forum de 2004 s'est tenu en Inde, à Mumbai. En 2005, le Forum est revenu à Porto Alegre, dont l'opposition avait conquis la mairie, tout en maintenant le budget de participation au Forum, au nom de son impact et de sa légitimité locale. Le conseil du FSM a repris ensuite sa politique de mobilité, avec en 2006 des réunions simultanées en Amérique latine (Caracas au Venezuela) et en Afrique (Bamako au Mali). En 2007, c'est Nairobi, au Kenya, qui a accueilli le Forum. Toutes ces rencontres ont témoigné de l'ampleur des mobilisations (environ 150000 participants à chaque fois) et de la vitalité de la pensée altermondialiste dans les trois continents.

\section{TC : Et le Parti des travailleurs?}

H.T. : Aujourd'hui, le Parti des travailleurs joue un rôle plus marqué dans l'approfondissement de la démocratie brésilienne, que les libéraux n'ont pas su conduire dans le passé. Le gouvernement Lula a compris que pour consolider la démocratie il fallait combiner la croissance de l'économie, la priorité à l'éducation à tous niveaux, et des politiques visant à combattre l'inégalité sociale sans délai. La valorisation des conquêtes démocratiques et l'élargissement de la base politique et sociale du régime sont des éléments centraux de cette ligne politique dont Lula est devenu le symbole.

Si d'importants progrès ont été accomplis dans cette direction, il reste encore beaucoup à faire. Le second mandat présidentiel, appuyé sur une large coalition gouvernementale, en crée les conditions politiques. La stratégie est établie, et l'économie peut entrer dans une nouvelle phase de croissance et de progrès social. Tout dépend maintenant de la capacité de mettre en ouvre ce réformisme pragmatique, qui marque la maturité politique de Lula, une fois tirées les leçons du premier mandat. 
La ligne Lula combine certains éléments d'économie libérale avec des mesures de protection sociale. Plus qu'une politique socialiste de gauche, il conduit une transition «libérale-démocratique» dont le socle sociologique est bien différent de celui sur lequel se sont appuyés les libéraux brésiliens qui étaient, en général, des représentants d'une démocratie élitiste. Lula se rapproche ainsi d'une politique socialedémocrate, dans un pays marqué par une forte tradition capitaliste, appuyée par l’État dans la phase "développementaliste» de Vargas à Geisel. Dans la période d’hégémonie néo-libérale, l'Argentine de Menem, le Brésil de Cardoso et bien sûr le Mexique ont connu des stratégies économiques d'ouverture internationale, voire de dépendance. Le Mexique s'est le plus avancé dans cette direction en rejoignant la Zone de libre-échange des Amériques (ZLEA). Le Brésil et l'Argentine, pour leur part, ont amoindri le rôle de l’État dans l'économie par le biais de privatisations d'entreprises publiques. Mais le gouvernement Lula a refusé l'adhésion du Brésil à la ZLEA. Il a poursuivi la maîtrise de l'inflation et le remboursement de la dette extérieure, tout en définissant une stratégie d'expansion de l'économie appuyée sur les potentialités du marché intérieur stimulées par des plans coordonnés par l'État, et sur une politique d'insertion internationale «souveraine» dans la globalisation.

TC : On peut donc dire que la réélection de Lula marque l'adhésion populaire à la voie sociale-démocrate, alors même que le Parti des travailleurs s'est divisé face à sa politique économique jugée trop orthodoxe par une fraction du parti?

H. T. : L'érosion politique du Parti des travailleurs fut largement provoquée par la corruption de certains membres du gouvernement, tandis que le succès de la politique économique conduite par Lula apparaissait bien improbable à ses adversaires, après les deux mandats du président Cardoso qui avait mis en place le "plan Real » pour combattre l'inflation. La critique de la gauche formulée au sein du Parti des travailleurs a conduit à des expulsions de certains parlementaires. C'est dans ce contexte qu'a été formé, en 2004, le Parti du socialisme et de la liberté (PSoL), qui reste un petit parti de d'extrême gauche.

Malgré ces critiques de gauche, malgré la corruption, et en dépit du choix d'une politique continue de stabilisation financière, Lula a pu rétablir sa popularité en raison des résultats de la politique sociale de son gouvernement. Une majorité des électeurs a considéré que l'ascenseur social fonctionne pour une partie de la population pauvre, et l'augmentation du pouvoir d'achat a fait entrer dans l'économie de marché des secteurs qui en étaient exclus. L'extraordinaire victoire que fut en 2006 la réélection de Lula, avec plus de $60 \%$ des suffrages exprimés, tient à cette combinaison entre une économie gérée sans crise par un ancien syndicaliste charismatique, et les effets concrets des politiques sociales qui ont conforté la légitimité politique du président sortant. L'échec de son adversaire, l'ancien gouverneur de São Paulo Geraldo Alckmin, témoigne aussi de la forte dépréciation des politiques néo-libérales héritées de Fernando Henrique Cardoso. Quelles que soient les continuités entre Cardoso et Lula, le principal atout de la politique économique de Lula a été de résorber la crise économique et financière laissée par Cardoso, et d'avoir jugulé le risque réel d'un retour de l'inflation. 
TC : Peut-on dire que le Brésil est un cas à part, en raison de son importance démographique et économique, et de sa taille? Comment ce pays émergent de grand poids est-il vu par ses voisins : comme inévitablement hégémonique ou comme un moteur politique et économique pouvant entraîner toute l'Amérique latine?

H.T. : L'unité attribuée par certaines visions idéalisées du passé, centrées sur le concept d'«Amérique latine», pèse aujourd'hui beaucoup moins lourd dans l'ensemble du continent latino-américain. En dépit de l'histoire commune des colonies des métropoles de la péninsule ibérique, la dynamique des nouveaux pays issus de l'Amérique espagnole a été, depuis leur indépendance dans la première moitié du XIX siècle, fort différente de celle du Brésil.

La fragmentation du territoire résultant des guerres de l'indépendance et de l'adoption de multiples régimes républicains contraste, dans cette région qu'Alain Rouquié a appelée l'« Extrême-Occident», avec l'unité territoriale assurée par l'élite politique civile brésilienne au fil d'une longue période monarchique. Bien sûr, l'extension du territoire fut également un élément important dans les rapports du Brésil avec d'autres pays de la région. Les seuls pays qui ont pu entrer en compétition avec lui en matière d'hégémonie ont été l'Argentine jusqu'aux années 1950 - en raison de la croissance de son économie associée à l'Angleterre - et le Mexique, stimulé par la révolution sociale de 1910 qui, sous la longue domination du Parti révolutionnaire institutionnel, a donné naissance à une économie nationale assez dynamique. En termes de PNB, l'économie mexicaine est aujourd'hui plus puissante que celle du Brésil, mais son impact est amenuisé par sa dépendance envers les États-Unis. Quant à l'Argentine, elle a perdu de son poids traditionnel en raison des crises économiques et financières prolongées qu'elle a connues. Son récent redressement ne lui a pas encore permis d'assumer une position éminente à l'échelle continentale.

Dans le Mercosur, l'économie brésilienne bénéficie d'une position hégémonique par rapport à ses partenaires. L'économie chilienne est certainement la plus stable. Elle connaît l'un des taux de croissance les plus élevés de la région. Mais le pays, relativement peu peuplé qui plus est, regarde volontiers vers le Pacifique, hors de l'Amérique latine. À l'inverse, le Brésil, à la recherche de nouveaux partenaires énergétiques dans le domaine du pétrole et du gaz naturel, conduit une active politique extérieure de voisinage, vis-à-vis du Venezuela, de la Bolivie, du Pérou, de l'Équateur. Dans ce contexte, le Brésil est bien un pays-clé dans l'équilibre politique et économique de l'Amérique latine.

TC : Dans de multiples pays - l'Équateur en 1997, en 2000, en 2005, l'Argentine en 2001, la Bolivie en 2003 - des présidents élus ont dû quitter le pouvoir face à la pression de la rue. Comment interpréter ces événements? Faut-il y voir la marque d'une phase de transition inachevée vers la démocratie représentative des assemblées, ou plutôt le contrecoup d'échecs économiques?

H.T. : La consolidation de la démocratie en Amérique latine est fortement liée à la réussite des politiques macroéconomiques. L'Argentine en est un cas exemplaire. La victoire de Carlos Menem à la succession de Raúl Alfonsín, en 1989, est 
due à l'échec du "plan austral » mis en place pour lutter contre l'hyper-inflation. De même, l'échec de Menem en 1999, au terme de son second mandat, doit beaucoup à la poussée inflationniste et aux contrecoups de sa politique de privatisations. Deux ans plus tard, l'approfondissement de la crise économique mène à la mobilisation populaire des piqueteros, qui fit tomber Fernando de la Rúa.

Quelques années auparavant, au Pérou, la pression de la rue avait chassé Alberto Fujimori du pouvoir. Comptaient certes les scandales de corruption de son gouvernement et sa tentative d'obtenir un troisième mandat contraire à la Constitution. Mais sur le fond, il avait instauré un "populisme de droite» qui, à la différence des populismes de gauche de Perón et de Vargas, n’avait rien redistribué au peuple marginalisé par la crise prolongée de la «décennie perdue» des années 1980.

Il faut se rappeler enfin que dans certains pays (le Brésil et le Chili) les dictatures militaires ont connu des phases de croissance, voire de "miracle économique», malgré la brutalité de la répression, beaucoup plus dure au Chili qu'au Brésil d'ailleurs. Le retour des régimes démocratiques, quant à lui, n'a pas toujours satisfait les attentes économiques et sociales qu'il avait fait naître. Les résultats ont été inégaux. Au Chili, les gouvernements successifs de la "Concertation» ont suscité le dynamisme économique le plus marqué de l'Amérique latine. Dans les années 1980, l’Argentine et le Brésil ont connu des temps difficiles avec l'échec des plans de stabilisation de Raúl Alfonsín et de José Sarney. Le "plan Real» conçu par Cardoso, alors ministre des Finances du gouvernement d'Itamar Franco, parvenu au pouvoir après la destitution de Collor en 1992, a ramené la stabilité financière pendant une décennie, alors que l'Argentine a subi la crise économique la plus grave de son histoire, et n'en sort que peu à peu sous le gouvernement Kirchner. Après la crise qui a marqué la fin du second mandat de Cardoso, Lula a repris une politique de stabilisation financière. Il encourage les exportations et les investissements étrangers. Le principal défi de son second mandant est bien de relancer la croissance, perçue comme un outil de consolidation démocratique, dès lors qu'elle s'accompagne d'une politique sociale.

TC : Comment faut-il interpréter la récente tournée conduite par GeorgeW. Bush au Brésil, en Uruguay, en Colombie, au Guatemala et au Mexique, en mars 2007?

H.T. : La visite de Bush en Amérique latine a cherché à rehausser, aux États-Unis même, la popularité de l'administration républicaine, plus affaiblie encore après la victoire démocrate aux élections législatives de novembre 2006. Mais elle avait aussi trois autres objectifs. Le premier, rendre visite à des pays ayant des gouvernements idéologiquement proches de Washington. La Colombie, qui a demandé l'appui des États-Unis dans le combat engagé contre la drogue et contre la guérilla de la FARC (les Forces armées révolutionnaires colombiennes), est devenu un pays stratégique pour assurer la présence de l'armée américaine en Amérique latine après les attentats terroristes du 11 septembre. Quant au Mexique, le grand voisin du Sud dont les dernières élections présidentielles, contestées par l'opposition de gauche, ont divisé le pays, il s'agissait d'y marquer le soutien américain au président Felipe Calderón, tout en abordant la question de l'immigration clandestine qui reste un problème permanent entre les deux pays. 
Le second objectif de la visite de Bush était d'améliorer les relations avec des pays comme le Brésil de Lula et l'Uruguay de Tabaré Vázquez, où les gouvernements, légitimés par leur large soutien populaire, cherchent certes à dépasser les politiques néo-libérales, mais apparaissent moins imprévisibles que ceux d'Hugo Chávez au Venezuela ou d'Evo Morales en Bolivie. Le troisième objectif était en réalité le plus important: discuter avec le Brésil de la technologie de l'éthanol capable de créer des combustibles alternatifs, à partir de la canne à sucre. Il s'agissait à la fois de répondre aux pressions démocrates accusant l'administration Bush de ne rien faire contre la menace croissante du réchauffement climatique, et de préparer avec le Brésil des accords à long terme dans le domaine énergétique. Notons au passage que c'est la première fois qu'une technologie développée en Amérique latine intéresse si fortement les États-Unis.

TC : Quels sont, à l'échelle mondiale, les enjeux que porte l'Amérique latine? L'Asie émergente, avec la poussée parallèle de l'Inde et de la Chine, se positionne en tant que future puissance, économique et stratégique. L'Afrique reste encore handicapée par ses crises, même si certains pays ont un parcours prometteur. La Russie réaffirme ses ambitions sous Poutine, avec un État qui réintervient dans l'économie. L'Union européenne reste un puissance économique majeure et un acteur diplomatique non négligeable, mais elle est politiquement en panne, à l'heure de son cinquantième anniversaire. Les États-Unis commencent à s'interroger sur ce que pourrait être une diplomatie post-Bush, moins unilatéraliste. Dans ce tableau à grands traits de l'ordre géopolitique mondial, y a-t-il une vision dominante en Amérique latine sur l'avenir du continent, quelles que soient les divergences existant aujourd'hui entre les lignes politiques des divers pays latino-américains?

H.T. : La question est trop complexe pour qu'on puisse y répondre en termes généraux pour l'ensemble de l'Amérique latine. La présence agressive de la Chine dans le champ commercial international et dans la compétition économique mondiale est un fait global majeur, qui a de fortes répercussions sur l'Amérique latine, où les entreprises chinoises multiplient les initiatives. Le Mercosur, de son côté, a privilégié les relations avec l'Union européenne, jusqu'à ce que les urgences d'un rééquilibrage interne dû à l'élargissement de sa base territoriale affaiblissent, momentanément sans doute, le dynamisme de sa politique de coopération internationale. Quant au Mexique, à la Colombie, au Chili et plus récemment à l'Uruguay, ils ont signé des accords privilégiés avec les États-Unis. Le Venezuela, grand pays pétrolier, a des rapports réguliers avec les pays arabes, et l'Argentine reste un partenaire important de l'Espagne. En fait, en matière de commerce international, la plupart des pays exportateurs visent plusieurs marchés sectoriels : les États-Unis pour le pétrole; l'Europe et les États-Unis pour la viande bovine; les pays arabes pour les volailles, l'Europe pour le soja et les fruits, la Chine, de plus en plus, pour les matières premières.

Dans ce contexte, le Brésil est le pays qui a plus diversifié ses exportations, vers l'Amérique latine elle-même, vers l'Asie et vers l'Afrique. La puissance de l'agriculture et de l'industrie brésiliennes, en dépit de taux de croissance plus faibles que 
ceux de la Chine, de l'Inde ou du Mexique, donne au Brésil un rôle de leadership dans les négociations conduites au sein de l'Organisation mondiale du commerce, souvent en alliance avec l'Inde et la Chine, car ces pays émergents ont des intérêts communs sur le plan du commerce international. De plus, la taille du marché interne de pays tels que le Brésil, la Russie, l'Inde, la Chine (ceux qu'on appelle les BRIC) est un facteur important pour le développement de leur économie. Certes, la stratégie du gouvernement Lula de diversification des relations internationales donne au Brésil un poids important en Amérique latine, mais chaque pays essaie de mettre en place la stratégie qui lui est la plus favorable. Ainsi les accords et les investissements du Venezuela d'Hugo Chávez en Amérique latine représentent-ils aujourd'hui le double de l'aide américaine consentie pour l'ensemble du continent. C'est sans doute en ce domaine que se trouve le vrai clivage entre le leadership de Lula et celui de Chávez en Amérique latine.

Entretien conduit par Jean-Luc Racine 